\title{
ASC0 2018: Fortgeschrittenes Mammakarzinom - CDK4/6-Inhibitoren in praktisch allen Situationen wirksam
}

Mit dem diesjährigen ASCO-Jahreskongress wurde verdeutlicht, dass die CDK4/6-Inhibitoren beim Hormonrezeptor-positiven, HER2-negativen metastasierten Mammakarzinom sowohl in der Erstlinie als auch in späteren Therapielinien, bei prä- und postmenopausalen Patientinnen, in der endokrin sensitiven und resistenten Situation sowie mit jedem endokrinen Kombinationspartner etwa gleich gut wirksam sind. Sie sollten daher immer zusätzlich zur endokrinen Therapie eingesetzt werden.

\section{MONALEESA-3: Ribociclib/Fulvestrant bei de novo und rezidivierter Erkrankung}

Bereits in den Phase-III-Studien PALOMA-3 und MONARCH-2 wurde Fulvestrant als endokriner Kombinationspartner mit den CDK4/6-Inhibitoren Palbociclib bzw. Abemaciclib erfolgreich getestet. Jetzt präsentierte Dennis Slamon aus Santa Monica, USA, die noch fehlenden Phase-III-Daten zur Kombination mit Fulvestrant und dem dritten CDK4/6-Inhibitor Ribociclib aus der MONALEESA-3-Studie [1]. In diese Studie wurden 726 postmenopausale Frauen mit fortgeschrittenem Hormonrezeptor-positivem, HER2-negativem Brustkrebs eingeschlossen. In einer 2:1 Randomisierung wurden 484 von ihnen mit Fulvestrant plus Ribociclib behandelt und 242 erhielten Placebo plus Fulvestrant. Die Studienpopulation umfasste sowohl Patientinnen, bei denen es während einer endokrinen Therapie in der metastasierten Situation zum Rezidiv bzw. innerhalb von 12 Monaten nach abgeschlossener adjuvanten Therapie zum Progress kam (Zweitliniensetting), als auch Patientinnen mit de novo Erkrankung (Erstliniensetting).

Ribociclib verlängerte in Kombination mit Fulvestrant das progressionsfreie Überleben (PFS) signifikant auf 20,5 Monate im Vergleich zu 12,8 Monate unter Fulvestrant alleine und reduzierte so das Risiko für eine Progression um 41\% (Hazard Ratio (HR): 0,59; $\mathrm{p}=0,0000004)$. Der deutliche PFS-Vorteil zeigte sich sowohl im Erstliniensetting (nicht erreicht vs. 18,3 Monate; HR: 0,58) als auch im Zweitliniensetting (14,6 vs. 9,1 Monate; HR: 0,57). Alle untersuchten Subgruppen profitierten; inklusive Patientinnen mit Metastasen in Lunge oder Leber (HR: 0,65). Ribociclib verbesserte auch die Gesamtansprechrate signifikant, in der Gesamtkohorte von 21,5 auf $32,4 \%(\mathrm{p}=0,00091)$ und bei den Frauen mit messbarer Krankheit von 28,7 auf 40,9 \% ( $\mathrm{p}=0,003)$. Die Daten zum Gesamtüberleben waren zum Zeitpunkt der Analyse noch unreif, im Ribociclib-Arm waren 14,5\% der Patientinnen verstorben und im Placebo-Arm 20,7 \%. Der CDK4/6-Inhhibitor konnte sicher mit Fulvestrant kombiniert werden mit den für Ribociclib bekannten und beherrschbaren Nebenwirkungen. Damit stellt Ribociclib in Kombination mit Fulvestrant eine neue Erst- und Zweitlinienoption für postmenopausale Frauen mit Hormonrezeptor-positivem, HER2-negativem fortgeschrittenem Mammakarzinom dar.

\section{MONARCH-2: Abemaciclib/Fulvestrant auch bei} prämenopausalen Frauen effektiv

Bei der ASCO-Jahrestagung 2017 gab es die ersten positiven Phase-III-Daten der MONARCH-2-Studie mit Abemaciclib in Kombination mit Fulvestrant [2]. Zur Erinnerung: In der Studie wurden 669 endokrin resistente Patientinnen mit Hormonrezeptor-positivem, HER2-negativem fortgeschrittenem Mammakarzinom in zwei Studienarme im Verhältnis 2:1 randomisiert. Die Abemaciclib-Dosierung wurde im Therapieverlauf (nach 178 Patientinnen) von $200 \mathrm{mg}$ auf $150 \mathrm{mg}$ zweimal täglich reduziert, nachdem vermehrt Diarrhöen aufgetreten waren. Die Zugabe von Abemaciclib zu Fulvestrant verlängerte das PFS signifikant gegenüber Fulvestrant alleine, von 9,3 auf 16,4 Monate (HR: 0,55; $\mathrm{p}<0,0000001)$. Die Ansprechrate wurde ebenfalls signifikant von 21,3 auf $48,1 \%$ mehr als verdoppelt. Erfreulich war, dass auch prämenopausale Frauen ebenso profitierten wie die gesamte Studienpopulation, da diese Patientengruppe immerhin etwa 35\% der Patientinnen ausmacht und deren Erkrankung oft einen aggressiveren Verlauf nimmt. Für diese Patientinnen sind zudem die endokrinen Therapieoptionen limitiert. Patrick Neven aus Leuven, Belgien präsentierte auf dem diesjährigen ASCO eine Detailanalyse der 117 prä- und perimenopausalen Frauen aus der MONARCH2-Studie [3]. Bei ihnen war das mediane PFS unter Abemaciclib plus Fulvestrant (plus GnRH-Agonist zur ovariellen Suppression)

\section{KARGER}

() 2018 S. Karger GmbH, Freiburg 
noch nicht erreicht, während es unter Placebo plus Fulvestrant (plus GnRH-Agonist) bei 10,5 Monaten lag (HR: 0,45; $\mathrm{p}=0,002$ ). Signifikant verbessert war auch die objektive Gesamtansprechrate ( 60,8 vs. $28,6 \%$ ). Hinsichtlich des Nebenwirkungsprofils unterscheidet sich Abemaciclib etwas von den beiden anderen CDK4/6-
Inhibitoren Palbociclib und Ribociclib, mit einer vergleichsweise geringeren Hämatotoxizität, aber einer deutlich höheren gastrointestinalen Toxizität, insbesondere Diarrhö. Die zusätzliche Gabe eines GnRH-Agonisten erhöhte die Toxizität der Behandlung nicht.

Dr. med. Gerhard Emrich

\section{References}

1 Slamon DJ et al.: Ribociclib (RIB) + fulvestrant (FUL) in postmenopausal women with hormone receptorpositive (HR+), HER2-negative (HER2-) advanced breast cancer $(\mathrm{ABC})$ : Results from MONALEESA-3. ASCO 2018, Abstr. 1000.
2 Sledge GW et al.: MONARCH 2: Abemaciclib in combination with fulvestrant in patients with HR+/HER2advanced breast cancer who progressed on endocrine therapy. ASCO 2017, Abstr. 1000.
3 Neven $\mathrm{P}$ et al.: Abemaciclib for pre/perimenopausal women with HR+, HER2- advanced breast cancer. ASCO 2018, Abstr. 1002. 